\title{
Revertant mosaicism: partial correction of a germ-line mutation in COL17A1 by a frame-restoring mutation
}

\author{
Thomas N. Darling, ${ }^{1}$ Carole Yee, ${ }^{1}$ Johann W. Bauer, ${ }^{2}$ Helmut Hintner, ${ }^{2}$ \\ and Kim B. Yancey ${ }^{1}$ \\ ${ }^{1}$ Dermatology Branch, National Cancer Institute, National Institutes of Health, Bethesda, Maryland 20892-1908, USA
${ }^{2}$ Department of Dermatology, General Hospital, A-5020 Salzburg, Austria
}

Address correspondence to: Thomas N. Darling, Dermatology Branch, National Cancer Institute, National Institutes of Health, Building 10, Room 12N238, 10 Center Drive MSC 1908, Bethesda, Maryland 20892-1908, USA. Phone: (301) 402-1863; Fax: (301) 496-5370; E-mail: tnd@box-t.nih.gov.

Received for publication June 18, 1998, and accepted in revised form April 16, 1999.

Generalized atrophic benign epidermolysis bullosa is an autosomal recessive subepidermal blistering disease typified by null mutations in COL17A1. In 1 large kindred, affected individuals were homozygous for a 2-bp deletion in COL17A1, 4003delTC, which resulted in a downstream premature termination codon, nonsense-mediated mRNA decay, and abrogation of type XVII collagen synthesis. Interestingly, 1 of these patients, although phenotypically identical to her affected siblings, showed focal expression of type XVII collagen in epidermal basement membrane in a pattern suggestive of revertant mosaicism. When studies of randomly obtained epidermal, oromucosal, and peripheral blood cells failed to identify the genetic basis of this apparent mosaicism, microscopic subpopulations of potentially revertant epidermal cells (i.e., those overlying basement membrane containing type XVII collagen) were selectively isolated using laser capture microdissection. Analysis of DNA and RNA from these cells revealed a second mutation, 4080insGG, on 1 allele of COL17A1. This 2-bp insertion corrected the reading frame just proximal to the premature termination codon, countered nonsense-mediated mRNA decay, and allowed protein production by patient keratinocytes in vivo and in vitro. These studies elucidate the molecular basis of a novel form of revertant mosaicism in humans.

J. Clin. Invest. 103:1371-1377 (1999).

\section{Introduction}

Genetic mosaicism is the presence of 2 or more genetically different cell populations within an individual. These cell populations can arise after fertilization as a consequence of large chromosomal abnormalities or mutations in single genes (1-3). Classically, such genetic events convert normal cells to mutant cells. In a recently documented form of mosaicism called revertant mosaicism (4), mutant cells undergo a second genetic event that restores protein function or expression, generating normal-appearing cells on a background of mutant cells. Although the occurrence of such revertant cells has been reported in multiple patients with Duchenne muscular dystrophy (5), tyrosinemia type I (6), Fanconi's anemia (7), Bloom's syndrome (8), and generalized atrophic benign epidermolysis bullosa (GABEB) (4, 9-13), the molecular basis of this mosaicism has been defined only rarely.

GABEB (no. 226650, Online Mendelian Inheritance in Man, http://www.ncbi.nlm.nih.gov/Omim) is an autosomal recessive chronic subepidermal blistering disease of skin and mucous membranes (14). Immunofluorescence (IF) microscopy studies by several groups found that type XVII collagen, a transmembrane hemidesmosomal protein encoded by a single copy gene, COL17A1, is not expressed in the epidermal basement membrane (BM) of most GABEB patients $(9,10,15,16)$. The central role played by this protein in the pathogenesis of GABEB was reinforced by the identification of mutations in COL17A1 in a number of patients with this inherited blistering disease (summarized in ref. 14). In the largest GABEB kindred identified to date, affected individuals are homozygous for a 2-bp deletion, 4003 delTC, which results in a frameshift and a premature termination codon (PTC) 86 bp downstream $(17,18)$. This mutation results in nonsense-mediated mRNA decay that abolishes synthesis of type XVII collagen and accounts for the absence of this adhesion molecule in the epidermal BM of these patients (18). Interestingly, 1 affected individual in this large kindred showed focal areas of epidermal BM that stained positive for type XVII collagen by IF microscopy (9), suggesting revertant mosaicism as described above.

In the present study, the genetic basis for revertant mosaicism in this GABEB patient was identified using laser capture microdissection (LCM). This recently developed technique $(19,20)$ permitted the selective isolation of epidermal cells overlying regions of BM that stain positive for type XVII collagen and was instrumental in identifying the genetic event responsible for immunoreactive protein in this patient's skin. We show that this patient, homozygous for the germ-line deletion 4003delTC, was mosaic for a unique frame-restoring mutation (4080insGG) on 1 allele. The second mutation eliminated the downstream PTC, countered nonsense-mediated mRNA decay, and resulted in measurable levels of this double-mutant transcript. Although this partial correction resulted in production of protein of appropriate immunoreactivity and size, it was deduced to contain 25 incorrect amino acids 
encoded by the shifted reading frame between the deletion and insertion. These studies elucidate the molecular basis of a novel form of revertant mosaicism in humans, namely mosaic partial correction of a germ-line deletion by a second, frame-restoring mutation.

\section{Methods}

Kindred. The proband, a 56-year-old woman, is a member of a large Austrian GABEB kindred that includes 5 affected and 5 unaffected siblings in 1 generation, as well as a pedigree that signifies propagation of the mutant allele through at least 6 generations $(18,21)$. All 4 living affected siblings ( 1 affected having died in infancy because of complications of this inherited blistering disease) are homozygous for a 2-bp deletion in COL17A1, 4003delTC, and show the same homozygous haplotype for 5 intragenic COL17A1 polymorphisms $(17,18,22)$. The proband's skin shows the same extent and character of blistering as that of her affected siblings; i.e., regions of nonfragile skin are not present.

Tissue. Five skin biopsies ( $4 \mathrm{~mm}$ in diameter) were obtained from the proband; all were from nonblistered skin. Three of the biopsies ( 1 from the left lower back, 2 from the right upper arm) were used for IF microscopy and LCM; 2 biopsies (from the left shoulder and forearm) were obtained to yield keratinocytes for culture. As controls, 3-mm skin biopsies were obtained from the left upper arm of a normal volunteer and the right upper arm of the proband's unaffected sister, an individual known to be heterozygous for 4003 delTC. Buccal mucosal brushings and peripheral blood samples were obtained from the proband, as well as from a normal volunteer, and were processed for analysis of genomic DNA.

Keratinocyte cultures. Two skin biopsies from the proband were immediately placed in serum-free media (Keratinocyte-SFM; GIBCO BRL, Rockville, Maryland, USA) and kept at $4^{\circ} \mathrm{C}$. Epidermal cell suspensions were created with $0.25 \%$ trypsin, and keratinocytes were cultured as described (18). Keratinocytes cultured from the foreskins of healthy newborns served as controls.

IF microscopy. All skin biopsies obtained for IF microscopy (and LCM) were immediately placed in Tissue-Tek OCT Compound (Miles Inc., Elkhart, Indiana, USA), frozen in liquid nitrogen, and stored at $-70^{\circ} \mathrm{C}$. Eight-micrometer cryosections of skin from the proband (and a normal volunteer) were studied by IF microscopy as described $(9,23)$. Anti-type XVII collagen antibodies used in mapping studies (i.e., experiments aimed at identifying sites in the proband's epidermal BM that contained or lacked type XVII collagen) included a murine mAb (HD18; a gift of M. Liebert, University of Texas, Houston, Texas, USA, and G. Giudice, Medical College of Wisconsin, Milwaukee, Wisconsin, USA) or rabbit antiserum developed against a baculovirusencoded recombinant form of type XVII collagen (23); secondstep antibodies were FITC-conjugated goat $\mathrm{F}\left(\mathrm{ab}^{\prime}\right)_{2}$ anti-mouse IgG (BioSource International, Camarillo, California, USA) (1:80) or FITC-conjugated goat $\mathrm{F}\left(\mathrm{ab}^{\prime}\right)_{2}$ anti-rabbit IgG (TAGO Inc., Burlingame, California, USA) (1:80). Double-staining IF microscopy experiments employed HD18 (1:20), biotinylated sheep anti-mouse IgG (Amersham Life Sciences Inc., Arlington Heights, Illinois, USA) (1:100), Texas Red streptavidin (Amersham Life Sciences Inc.) (1:50), rabbit anti-type IV collagen antiserum (originally developed and provided by G. Martin, National Institute of Dental Research, Bethesda, Maryland, USA) (1:20), and FITC-conjugated swine anti-rabbit IgG (DAKO Corp., Carpinteria, California, USA) (1:20).

LCM. Skin cryosections used for LCM were handled in 2 ways. For recovery of DNA, cryosections were incubated sequentially with HD18 and FITC-conjugated goat $\mathrm{F}\left(\mathrm{ab}^{\prime}\right)_{2}$ anti-mouse IgG, washed, overlaid with a coverslip, and then viewed by IF microscopy to identify sites of epidermal BM that stained either positive or negative for type XVII collagen. Coverslips were removed, and mapped skin sections were fixed in ethanol and stained with hematoxylin and eosin. A cap coated with a thermoplastic membrane was placed on top of these skin sections, which were examined with a light microscope fitted with a pulse-diode laser (Arcturus Engineering Inc., Mountain View, California, USA). Under direct observation, 40- to 60-mW pulses of the laser were applied to regions of epidermis overlying BM known to have stained either positive or negative for type XVII collagen - a treatment that specifically bound epidermal cells of interest to the cap $(19,20)$. The cap was lifted off the cryosection, and any nonspecifically adherent cells were tape-stripped from the membrane. The cap was placed on a standard microfuge tube containing extraction buffer, inverted, and then processed for analysis of genomic DNA (see below). For recovery of RNA, serial cryosections of skin were prepared and every third section was mapped by IF microscopy as described above. Intervening cryosections were immediately fixed in $70 \%$ ethanol, stained with hematoxylin and eosin, and subjected to LCM to recover epidermis overlying BM that contained or lacked type XVII collagen. Microdissected epidermis from 6-8 skin sections was collected on the same cap and used for analysis of epidermal RNA (see below).

DNA extraction. Microdissected epidermis from 1-3 skin sections was collected on the same cap and placed on a microfuge tube containing $20 \mu \mathrm{L}$ of $10 \mathrm{mM}$ Tris- $\mathrm{HCl}, 1 \mathrm{mM}$ EDTA, $1 \%$ Tween-20, and $0.1 \mathrm{mg} / \mathrm{mL}$ proteinase $\mathrm{K}$ ( $\mathrm{pH}$ 8.0). Tubes were inverted and incubated at $37^{\circ} \mathrm{C}$ overnight; proteinase $\mathrm{K}$ was inactivated by heating to $99.9^{\circ} \mathrm{C}$ for 10 minutes. Aliquots of the resulting mixture were used in PCR studies.

$R N A$ isolation. Caps containing microdissected epidermis were placed on RNase-free microfuge tubes holding $200 \mu \mathrm{L}$ denaturing solution and $1.6 \mu \mathrm{L} \beta$-mercaptoethanol, and were then processed to yield total RNA (Micro RNA Isolation Kit; Stratagene, La Jolla, California, USA). RNA was suspended in $9 \mu \mathrm{L}$ diethyl pyrocarbonate-treated water and $3 \mu \mathrm{L}$ RNase inhibitor (Perkin-Elmer Corp., Branchburg, New Jersey, USA) and then stored at $-70^{\circ} \mathrm{C}$.

Verification of 4003 delTC. PCR was used to amplify exon 52 (nucleotides 3872-4261) of the type XVII collagen gene (GenBank no. M91669) using primers based on the flanking intronic regions as described previously (17). The resulting 527-bp product was digested with NlaIII to demonstrate the presence or absence of the 4003delTC mutation (17). Allele-specific PCR was also used to detect $4003 \mathrm{delTC}$. These experiments used primers that end on sequences specific to the normal or mutant alleles at nucleotide 4003. The sense primers for the normal and the 4003delTC mutant sequences were 5'-GCTCCTCCTCACACAGCTCATC- 3 ' and 5'-GCTCCTCCTCACACAGCTCATG3', respectively. Cycling conditions for allele-specific PCR were the same as those for exon 52, except that the annealing temperature was $65^{\circ} \mathrm{C}$. The antisense primer for both of these allele-specific PCR studies was the intronic primer used to amplify exon 52, extended by 4 bp (5'-CCACAAACAAGAAAGCCAGTCTGG-3').

$R T-P C R$. To determine if exon 52 was skipped in the proband, aliquots of total RNA from her cultured keratinocytes were mixed with random hexamers and reverse transcribed to produce cDNAs. Resulting cDNAs were mixed with primers for COL17A1 (specifically, nucleotides 3853-3880 and 4610-4630; ref. 18) and amplified by PCR (GeneAmp RNA PCR; PerkinElmer Corp.) using 35 cycles of $95^{\circ} \mathrm{C}$ for 30 seconds, $56^{\circ} \mathrm{C}$ for 30 seconds, and $72^{\circ} \mathrm{C}$ for 60 seconds.

DNA sequencing. PCR products were sequenced (ABI Prism; Perkin-Elmer Corp.) directly or following cloning using a TOPO TA Cloning Kit (Invitrogen, San Diego, California, USA). Sequences of particular interest were analyzed with Sequencher 3.0 software (Gene Codes Corp., Ann Arbor, Michigan, USA). These studies identified 4080insGG, a 2-bp insertion that partially corrected 1 COL17A1 allele in the proband's revertant epidermal cells (see Results). 
Verification of 4080insGG. Allele-specific PCR was used to verify 4080insGG. The primers used for amplification of 4080insGG or the normal sequence at this site were $5^{\prime}$-CCTGGGTGCAGGCGGGGT-3' and 5'-CCTGGGTGCAGGCGGTGC-3', respectively; the annealing temperature for this reaction was $57^{\circ} \mathrm{C}$. The antisense primer was again the intronic primer used to amplify exon 52 , extended by $4 \mathrm{bp}$.

Analysis of COL17A1 transcripts. Following the identification of the frame-restoring mutation, 4080insGG, experiments were designed to assess the relative levels of COL17A1 transcripts derived from each allele in the proband's revertant epidermal cells, i.e., cells containing 14003 delTC allele and 1 allele that carried both 4003delTC and 4080insGG. For comparative purposes, analogous experiments assessed the relative levels of COL17A1 transcripts derived from each allele in a heterozygote, i.e., an unaffected sibling with 1 normal and 14003 delTC allele. In this manner, the relative stability of single-mutant (4003delTC) and double-mutant (4003delTC and 4080insGG) COL17A1 transcripts was examined, and the ability of the double-mutant allele to rescue COL17A1 transcripts from nonsense-mediated mRNA decay was explored. Two approaches were used to address this issue. In one set of experiments, LCM was used to collect the proband's revertant epidermal cells, as well as the epidermal cells of a sibling known to be heterozygous for 4003 delTC. Transcripts in these samples were reverse transcribed, and COL17A1 CDNAs were amplified by allele-specific PCR using the sense primers for normal and mutant sequences (as described above) combined with an antisense primer corresponding to nucleotides $4610-4630$ of the cDNA. The resulting PCR products were electrophoresed on 4-20\% polyacrylamide gels (Novex, San Diego, California, USA), stained with ethidium bromide, photographed, and quantitated by densitometric analysis of band intensity using the public domain NIH Image 1.60 program (developed at the NIH and available at http://rsb.info.nih.gov/nih-image/). In another set of experiments, revertant epidermal cells in the proband were isolated with LCM and processed to yield RNA. RNA was reverse transcribed as described above and PCR amplified using primers spanning exons 51-56 of COL17A1. Resulting cDNAs were cloned and analyzed by sequencing $(n=6)$ or allele-specific PCR $(n=19)$ to estimate the relative levels of transcripts derived from each COL17A1 allele.

Immunoprecipitation studies. Subconfluent monolayer cultures of keratinocytes derived from skin biopsies of the left forearm and shoulder of the proband (or from normal neonatal foreskins of controls) were biosynthetically radiolabeled with $\left[{ }^{35} \mathrm{~S}\right]$ methionine $(50 \mu \mathrm{Ci} / \mathrm{mL}$, specific activity $\sim 1,100 \mathrm{Ci} / \mathrm{mmol}$; Du Pont NEN Research Products, Boston, Massachusetts, USA) for 2 hours. Radiolabeled cell extracts were processed and studied by immunoprecipitation using rabbit antiserum against type XVII collagen (and controls) as described $(24,25)$.

\section{Results}

Type XVII collagen was present only at focal sites in the proband's epidermal BM. IF microscopy studies confirmed that monoclonal and polyclonal antibodies directed against type XVII collagen bound normal epidermal BM in a continuous manner (Figure 1a). In contrast, type XVII collagen was present in a focal, interrupted distribution in the proband's epidermal BM (Figure 1b). By comparison, type IV collagen, as well as other BM constituents (9), was present in the proband's skin (Figure 1c) in an amount and distribution identical to that seen in the skin of a normal volunteer (data not shown). Although this distribution of type XVII collagen was identified in IF microscopy studies of skin from both sides of the proband's body (specifically, the left lower back and the right upper arm), it was not possible to predict where such sites were located because there were no clinical findings (e.g., islands of normal-appearing skin) that indicated their existence. In fact, IF microscopy studies of a second biopsy from the proband's right
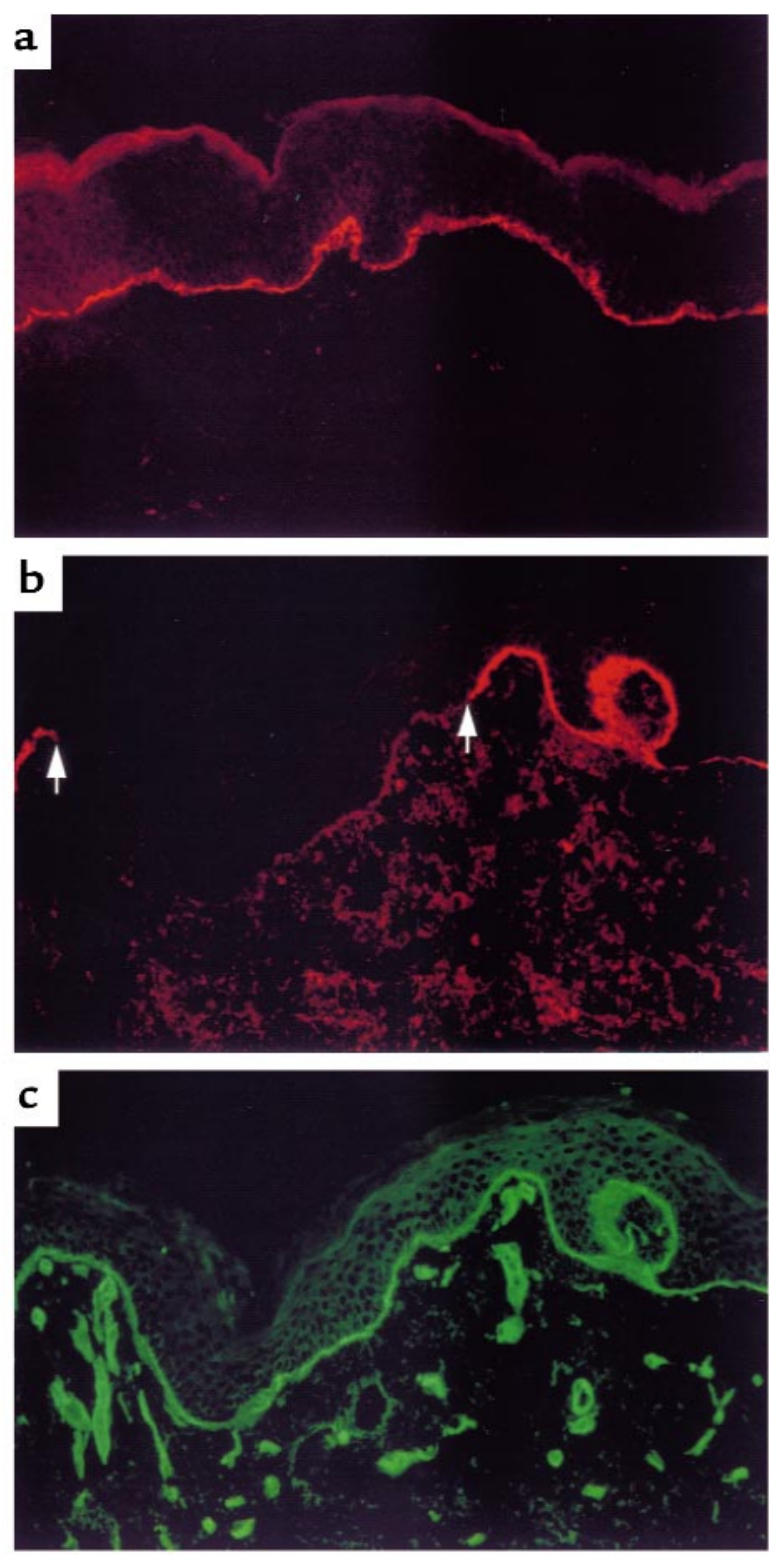

Figure 1

Type XVII collagen was present only at focal sites in the proband's epidermal BM. (a) IF microscopy studies of normal human skin cryosections stained with an mAb to type XVII collagen (see Methods) showed continuous expression of this protein in epidermal BM (shown in red). (b) IF microscopy studies of the proband's skin cryosections stained in the same manner found that type XVII collagen (when detected) was present in epidermal BM only in a focal, interrupted distribution (shown in red; an intervening segment of nonreactive BM is delineated by arrows). (c) The proband's epidermal BM was otherwise intact. For purposes of documentation, the cryosection shown in $\mathbf{b}$ was double stained with an antibody to type IV collagen. This antibody (as well as antibodies to bullous pemphigoid antigen 1, laminin 5, and type VII collagen; ref. 9) showed normal reactivity (shown in green) along the entire epidermal BM of the proband. Type IV collagen in microvascular BMs was also stained. 
a
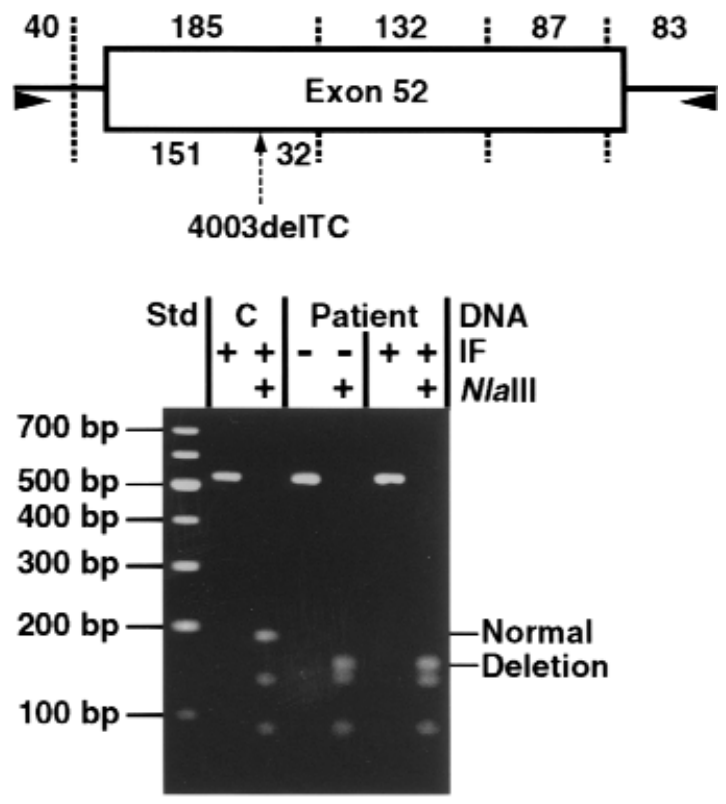

b

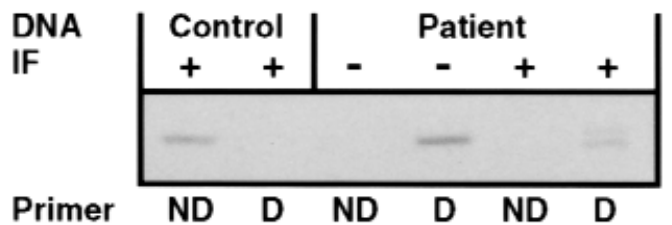

Figure 2

A normal COL17A1 allele was not present in the proband. (a) LCM was used to collect epidermal cells overlying segments of the proband's epidermal BM that stained positive (+) or negative (-) for type XVII collagen by IF microscopy (IF); epidermis collected from normal human skin by LCM served as a control (C). Genomic DNA was isolated from these samples, and exon 52 of COL17A1 was PCR amplified and analyzed by restriction endonuclease digestion. For illustration, the schematic depicts exon 52 (rectangle) and its flanking intronic regions (solid lines) that were amplified by PCR (primers indicated by arrowheads). Vertical dotted lines signify Nlalll restriction endonuclease sites in PCR products derived from a normal COL17A1 allele; the sizes of the resulting fragments (in base pairs) are listed along the top of the schematic. The arrow directed to the underside of exon 52 shows the Nlalll cleavage site introduced by 4003 delTC; this additional Nlall restriction site cleaves the 185-bp fragment into segments of 151 and 32 bp as indicated. The PCR products outlined above, and a 100-bp ladder standard (Std), were electrophoresed on a 3\% agarose gel and then stained with ethidium bromide. Undigested PCR products derived from control DNA, as well as negative-patient and positive-patient DNA, all comigrated as shown. Nlalll restriction analysis of these PCR products revealed that control DNA was derived from normal COL17A1 alleles and that both negative-patient and positive-patient DNA were derived from alleles bearing 4003delTC. Note that the 32- and 40-bp fragments are not apparent in this gel. (b) That positive-patient DNA was not derived from normal COL17A1 alleles was confirmed by allele-specific PCR using primers ending on sequences specific to the normal (ND, no deletion) or mutant (D, deletion) alleles at nucleotide 4003. Analysis of these PCR products on 4-20\% polyacrylamide gels confirmed the normal sequence in control DNA and showed that both negative-patient and positive-patient DNA were amplified from alleles containing 4003delTC. The presence of a doublet in the positive-patient (but not the negative-patient) DNA using the D primers suggested heterozygosity for a mutation downstream from 4003 delTC. upper arm found no immunoreactive type XVII collagen in any cryosection throughout the sample. The results of this study, taken together with prior studies, indicate that most skin biopsies from the proband contained no immunoreactive type XVII collagen; moreover, biopsies that were positive showed focal, interrupted expression of this protein in epidermal BM. Other affected family members, as well as other GABEB patients homozygous for 4003delTC, did not show focal, interrupted expression of type XVII collagen in their epidermal BMs $(9,18)$.

Studies of cultured keratinocytes, oral epithelial cells, and peripheral blood cells did not identify the molecular basis of the proband's apparent mosaicism. The serendipitous observation that the proband's epidermal BM showed focal, interrupted expression of type XVII collagen suggested that this individual was mosaic for either a normal COL17A1 allele or a second genetic alteration that allowed production of immunoreactive protein. To address the former possibility, we studied DNA isolated from the patient's peripheral blood, buccal mucosa, and cultured keratinocytes, using both NlaIII digestion of PCR-amplified exon 52 (the exon containing 4003delTC) and allele-specific PCR (see Methods). In brief, the normal COL17A1 allele was not identified in these studies. To address the second possibility, exon 52 was amplified from the DNA of cultured keratinocytes using intronspecific primers, and the PCR product was sequenced. The 4003delTC mutation was again demonstrated on both COL17A1 alleles, but a second genetic alteration was not identified. Given these findings, it was also considered that exon 52 might be completely deleted or skipped in the proband because loss of the 390 nucleotides in this exon would restore the normal reading frame in COL17A1. However, additional studies of genomic DNA (specifically, that spanning exons 51-53), as well as total RNA from this patient's cultured keratinocytes, showed no deletion of exon 52 from the COL17A1 gene or altered splicing of premRNA that led to skipping of this exon (data not shown). The failure of these studies to explain the focal expression of type XVII collagen in the proband's epidermal BM suggested that the underlying genetic alteration was either absent or insufficiently represented in the cell populations examined in these studies.

$L C M$ revealed that the proband's epidermis was mosaic for the frame-restoring mutation 4080ins GG. To enhance the possibility of detecting a genetic alteration expressed in a mosaic pattern, LCM was used to procure the proband's epidermal cells directly overlying segments of BM that contained or lacked type XVII collagen. DNA was extracted from these 2 populations of cells (hereafter referred to as positive- and negative-patient DNA, respectively) and compared with DNA extracted from laser-microdissected normal epidermis. Using intron-specific primers, exon 52 of COL17A1 was PCR amplified from these DNA samples and analyzed by NlaIII digestion. These studies demonstrated that both positive- and negative-patient DNA were derived solely from alleles containing 4003delTC and that DNA from control epidermis was derived from normal COL17A1 alleles (Figure 2a). As shown in Figure 2b, these results were confirmed by allele-specific PCR. Interestingly, PCR products obtained using primers to amplify 4003 delTC in positive-patient DNA showed a doublet when analyzed on 4-20\% polyacrylamide gels (Figure 2b), suggesting heterozygosity for a 
mutation downstream from 4003delTC.

To uncover a second mutation, the sequence of exon 52 in laser-microdissected positive-patient DNA was compared with that of DNA from laser-microdissected normal human epidermis (Figure 3a). A second mutation, 4080insGG, was detected on 1 COL17A1 allele of positivepatient DNA (Figure 3a). Cloning and sequencing showed either 4003delTC alone or 4003delTC in combination with 4080 ins GG (Figure $3 \mathrm{~b}$ ). To verify this mutation, primers were designed to amplify either $4080 \mathrm{ins} G \mathrm{G}$ or the sequence without the insertion ( $\mathrm{I}=$ insertion, $\mathrm{NI}=$ no insertion; Figure 4). Normal epidermal DNA and negative-patient DNA were amplified only with the NI primer, indicating absence of 4080insGG. Positive-patient DNA was amplified with both the I and NI primers, as expected for a sample heterozygous for the second mutation. When these primer pairs were used to reexamine DNA from prior samples of this patient's cultured keratinocytes and peripheral blood (i.e., samples tested earlier for the presence of a normal or corrected COL17A1 allele), 4080insGG was not found. These findings, as well as the complete absence of type XVII collagen in most skin biopsies, suggested that a minority of the proband's tissues were mosaic.

The frame-restoring mutation 4080insGG yields COL17A1 $m R N A$ and protein. The results described above indicated that the proband's revertant epidermal cells had 2 mutant COL17A1 alleles, one containing 4003delTC alone and one containing 4003delTC plus 4080insGG. The respective levels of transcripts derived from such single-mutant and double-mutant COL17A1 alleles in epidermal cells in vivo were examined in a series of experiments. Our hypotheses were (a) that transcripts derived from single-mutant alleles would be present in low levels because of nonsense-mediated mRNA decay (as shown in prior Northern blot studies examining total RNA from cultured keratinocytes of patients homozygous for 4003delTC; ref. 18); and (b) that transcripts derived from double-mutant alleles would exceed those derived from single-mutant alleles because of the elimination of the PTC by the "correcting mutation" in the former. To determine the relative level of transcripts derived from a single 4003delTC allele, LCM was used to recover epidermis from the proband's sister, who was known to be heterozygous for this mutation (18). Total RNA was then isolated, reverse transcribed, PCR amplified using primers specific for sequences that contained or lacked 4003delTC, and quantitated by densitometry. Separate experiments estimated that the ratio of normal to single-mutant transcripts in the epidermis of this heterozygote was approximately 30:1. To determine if 4080insGG in the proband's revertant epidermal cells yielded transcripts whose levels exceeded those derived from the allele bearing 4003delTC alone, a related series of experiments were performed. First, revertant epidermal cells in the proband were isolated by LCM and processed to yield total RNA. This RNA was reverse transcribed, PCR amplified using primers specific for sequences that contained or lacked 4080insGG, and quantitated by densitometry. Using this approach, separate experiments found that the double-mutant transcript was present at 2.5 times the level of transcripts containing 4003delTC alone. In other studies conducted in parallel, RNA from the proband's revertant epidermal cells was reverse transcribed and amplified a
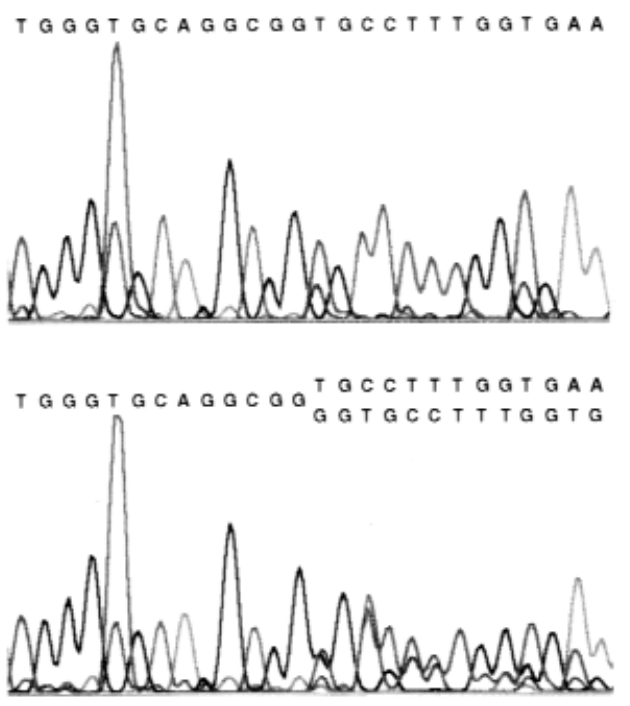

b
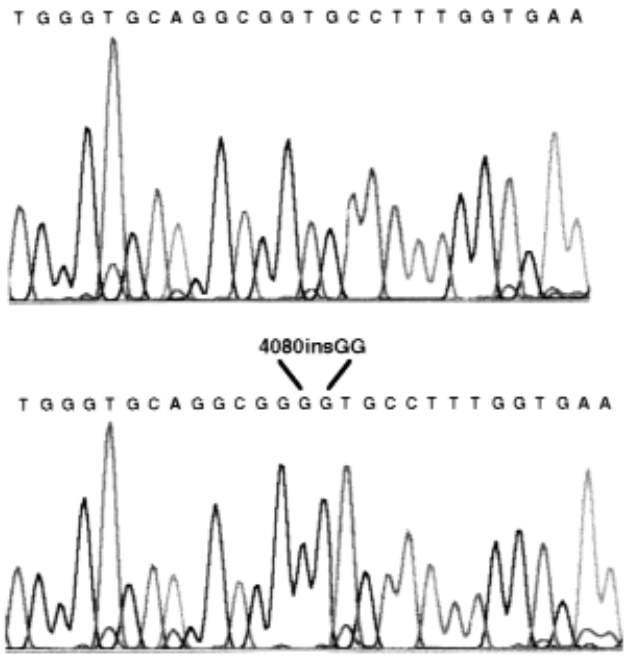

Figure 3

DNA from the proband's epidermis overlying BM containing type XVII collagen is homozygous for 4003delTC and heterozygous for a second COL17A1 mutation, 4080insGG. (a) Direct nucleotide sequencing of PCRamplified exon 52 from normal epidermis (top) shows the expected sequence. Patient epidermis overlying BM staining positive for type XVII collagen shows base ambiguities beginning at nucleotide 4080 in the CDNA (bottom). (b) Clones of the PCR product in a show either 4003 delTC, followed by the expected sequence (i.e., no insertion; top), or 4003delTC in combination with 4080insGG (bottom).

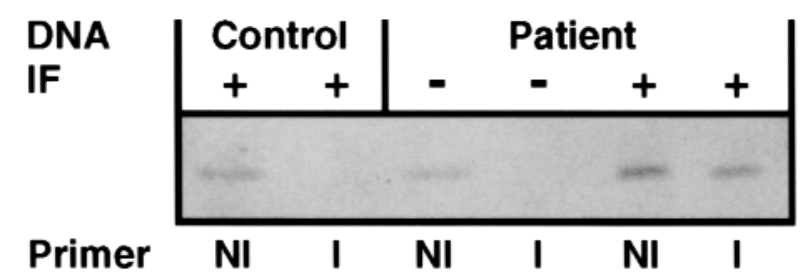

Figure 4

Verification of 4080insGG. PCR using primers that amplified either 4080insGG (I, insertion) or the allele without the insertion (NI) showed the presence of the former only in DNA from patient epidermis overlying BM containing type XVII collagen. Symbols are as designated in Figure 2. 


\section{Figure 5}

Immunoprecipitation studies of biosynthetically radiolabeled extracts from keratinocytes cultured from the proband or a normal control. (a) A rabbit antibody to a baculovirus-encoded recombinant of type XVII collagen precipitated a $180-\mathrm{kDa}$ protein in normal keratinocytes but not in the patient's keratinocytes (the latter procured from the proband's left forearm). (b) Studies of keratinocytes derived from the proband's left shoulder identified a 180kDa band that comigrated with type XVII collagen produced by normal keratinocytes. As a positive control, rabbit antibody to a bullous pemphigoid antigen 1 fusion protein (BPAG1) precipitated a 230$\mathrm{kDa}$ protein in all samples. No proteins were precipitated by preimmune rabbit serum. The exposure time was 6 and 10 days for $\mathbf{a}$ and $\mathbf{b}$, respectively. A 4-week exposure of a failed to detect type XVII collagen in the patient sample.

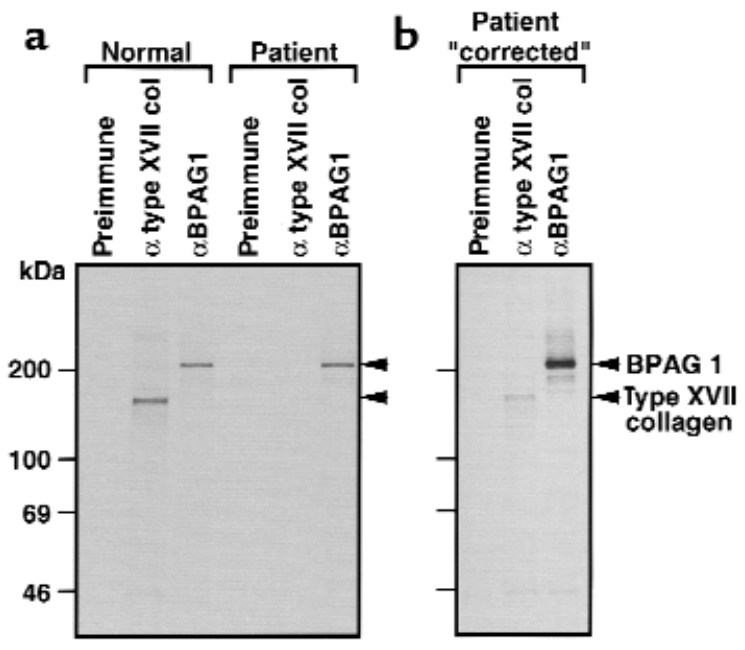

by PCR to yield cDNAs spanning exons 51-56. These cDNAs were cloned and analyzed to determine the frequencies of the double-mutant and single-mutant transcripts in 25 samples picked randomly. In these studies, the double-mutant cDNA was present in $84 \%$ of the samples (i.e., levels 5 times those of transcripts derived from 4003delTC alone). In sum, both of these approaches indicated that 4080insGG stabilized COL17A1 transcripts in the proband's revertant epidermal cells and countered nonsense-mediated mRNA decay that otherwise affects transcripts bearing 4003delTC.

To determine if this frame-restoring mutation resulted in the production of immunoreactive protein, keratinocytes from 2 biopsy sites on the proband were expanded in culture, biosynthetically radiolabeled, and studied by immunoprecipitation using a polyclonal antibody directed against type XVII collagen. Although keratinocytes from the left forearm did not produce type XVII collagen, those from the left shoulder synthesized a $180-\mathrm{kDa}$ protein that comigrated with type XVII collagen produced by normal keratinocytes (Figure 5). The ability to identify immunoreactive type XVII collagen in this set of keratinocytes was considered fortuitous, given the inability to predict which skin sites were "positive." These immunoprecipitation results corroborate IF microscopy studies indicating that some biopsies of this patient's skin were completely devoid of type XVII collagen, whereas others showed patchy expression of this protein (9).

\section{Discussion}

This report describes a type of revertant mosaicism not previously documented in humans, namely a partial gene correction by a frame-restoring mutation. As shown in this study, this correcting mutation restored the normal reading frame just prior to a PTC, countered nonsense-mediated mRNA decay, and led to the production of protein of appropriate immunoreactivity and size. Clinical and genetic findings in our patient contrast with those of another recently described GABEB patient with revertant mosaicism (4). The latter patient, a compound heterozygote for a paternal nonsense mutation and a maternal deletion, islands of normal-appearing skin clinically and restoration of a normal COL17A1 allele by mitotic gene conversion. This mechanism (as well as single and double crossing over) can account for revertant mosaicism in compound heterozygotes where germ-line mutations are located at different sites in a gene $(7,8)$. Different mechanisms are required to achieve revertant mosaicism in patients such as ours who are homozygous for a mutant allele.

Our initial hypothesis regarding the focal distribution of type XVII collagen in our patient's epidermal BM was that overlying keratinocytes had a back mutation that restored 1 COL17A1 allele to normal sequence. However, analysis of randomly acquired keratinocytes, oral mucosa, and peripheral blood cells, as well as revertant keratinocytes themselves, found no evidence of a normal COL17A1 allele. These studies also found that the reading frame of COL17A1 was not restored by in-frame deletion or skipping of exon 52 . Encouraged by the finding that subpopulations of epidermal cells could be selectively procured by LCM, we specifically examined genomic DNA from cells overlying segments of the proband's epidermal BM that contained type XVII collagen for evidence of a correcting mutation positioned between 4003delTC and the resulting PTC 86 bp downstream. The identification of 4080insGG, and the subsequent demonstration of the biologic consequences of this correcting mutation, elucidated the basis of this patient's revertant mosaicism. Analogous frame-restoring mutations have been observed experimentally in Saccharomyces cerevisiae (26). In brief, as shown by Greene and Jinks-Robertson (26), a +1-bp frameshift causing a PTC in the yeast LYS2 gene was compensated in almost all revertant colonies by a 1-bp deletion within the 150 nucleotides preceding the PTC, rather than by correction of the original genetic defect itself. Moreover, these 1-bp deletions tended to occur in homopolymer tracts, suggesting that slippage of the DNA polymerase accounted for these events. Interestingly, and in a corresponding manner, the 2-bp deletion in our GABEB patient was compensated by a 2-bp insertion that may also have originated as a consequence of DNA polymerase slippage, because 2 guanine nucleotides were reproduced at the site of the insertion. The timing of this 2-bp insertion during embryogenesis was not determined because it was not feasible to sample the proband's skin or other organs extensively to establish the distribution of this "correcting" mutation. However, its presence in epidermal cells (derived from 
the ectoderm) but not peripheral blood cells (derived from mesoderm) suggested that it occurred after primitive streak formation and the development of all 3 germ layers. To our knowledge, the basis of mosaicism in ectodermally derived keratinocytes is not different from that which occurs in cells of mesodermal or endodermal origin.

Mosaicism for the frame-restoring mutation resulted in focal, positive staining for type XVII collagen in the proband's epidermal BM. The protein, predicted to contain 25 incorrect amino acids encoded by the shifted reading frame between the deletion and the insertion, was deemed to be at least partially abnormal because skin resistant to blister induction was not present in the proband. The failure of the frame-restoring mutation to restore complete function is not unexpected because the substitution of even 1 amino acid in type XVII collagen can result in a blistering phenotype $(11,27)$. Had immunoreactive protein in this patient's epidermal BM not been observed serendipitously in our initial IF microscopy studies, this form of mosaicism would have gone unrecognized and unexplained. Interestingly, a similar finding has been reported in Duchenne muscular dystrophy, where as many as $50 \%$ of patients in some series have shown rare clusters of muscle fibers that stain positive for dystrophin $(5,28)$. In analogy to our patient, in whom the presence of immunoreactive protein in epidermal BM did not completely correct skin fragility, the presence of immunoreactive dystrophin in rare muscle fibers in patients with Duchenne muscular dystrophy has not been associated with a milder form of disease (28). Although the presence of such immunoreactive muscle fibers in patients with Duchenne muscular dystrophy was postulated to result from a second, frame-restoring mutation (5), such an event has not been documented previously. To our knowledge, our demonstration of a second, frame-restoring mutation in a revertant GABEB patient provides the first direct evidence for this mechanism and affords a potential explanation for cases of revertant mosaicism involving an initial, frameshift mutation. These findings vary from cases of revertant mosaicism where mutations are corrected by intragenic recombination or gene conversion - mechanisms documented in rare patients with tyrosinemia type I (6), Fanconi's anemia (7), or Bloom's syndrome (8). The elucidation of the genetic basis for corrected cells in GABEB and other diseases has been hampered by the inability to isolate unique and relevant subpopulations of cells from affected tissues. This study demonstrates the power and utility of LCM to procure genomic DNA and mRNA species from microscopic subpopulations of human epidermal cells. Moreover, it points out how even the most sophisticated testing methods are ineffective if the wrong cells are subjected to analysis. LCM clearly has direct relevance for the analysis of mosaicism, as well as for cell subpopulations in tissues undergoing morphogenesis, differentiation, or malignant transformation.

\section{Acknowledgments}

The authors are grateful to Mike Emmert-Buck and John Gillespie for their assistance in the LCM facility, to Zhengping Zhuang for thoughtful discussions, to Mark Udey and Jonathan Vogel for their review of this manuscript, and to Harry Schaefer for preparing figures.
1. Hall, J.G. 1988. Review and hypothesis. Somatic mosaicism: observations related to clinical genetics. Am. J. Hum. Genet. 43:355-363.

2. Happle, R. 1993. Mosaicism in human skin: understanding the patterns and mechanisms. Arch. Dermatol. 129:1460-1470.

3. Zlotogora, J. 1998. Germ line mosaicism. Hum. Genet. 102:381-386.

4. Jonkman, M.F., et al. 1997. Revertant mosaicism in epidermolysis bullosa caused by mitotic gene conversion. Cell. 88:543-551.

5. Klein, C.J., et al. 1992. Somatic reversion/suppression in Duchenne muscular dystrophy (DMD): evidence supporting a frame-restoring mechanism in rare dystrophin-positive fibers. Am. J. Hum. Genet. 50:950-959.

6. Kvittingen, E.A., Rootwelt, H., Berger, R., and Brandtzaeg, P. 1994. Selfinduced correction of the genetic defect in tyrosinemia type I. J. Clin. Invest. 94:1657-1661.

7. Lo Ten Foe, J.R., et al. 1997. Somatic mosaicism in Fanconi anemia: molecular basis and clinical significance. Eur. J. Hum. Genet. 5:137-148.

8. Ellis, N.A., et al. 1995. Somatic intragenic recombination within the mutated locus BLM can correct the high sister-chromatid exchange phenotype of Bloom syndrome cells. Am.J. Hum. Genet. 57:1019-1027.

9. Pohla-Gubo, G., et al. 1995. Diminished expression of the extracellular domain of bullous pemphigoid antigen 2 (BPAG2) in the epidermal basement membrane of patients with generalized atrophic benign epidermolysis bullosa. Exp. Dermatol. 4:199-206.

10. Jonkman, M.F., et al. 1996. Generalized atrophic benign epidermolysis bullosa: either the $180-\mathrm{kd}$ bullous pemphigoid antigen or laminin 5 is deficient. Arch. Dermatol. 132:145-150.

11. Schumann, H., et al. 1997. Three novel homozygous point mutations and a new polymorphism in the COL17A1 gene: relation to biological and clinical phenotypes of junctional epidermolysis bullosa. Am. J. Hum. Genet. 60:1344-1353.

12. Matsumura, Y., et al. 1997. Mosaic expression of uncein and 180-kDa bullous pemphigoid antigen in generalized atrophic benign epidermolysis bullosa. Br. J. Dermatol. 136:757-761.

13. Marinkovich, M.P., et al. 1997. LAD-1 is absent in a subset of junctional epidermolysis bullosa patients. J. Invest. Dermatol. 109:356-359.

14. Darling, T.N., Bauer, J.W., Hintner, H., and Yancey, K.B. 1998. Generalized atrophic benign epidermolysis bullosa. Adv. Dermatol. 13:87-119.

15. Jonkman, M.F., et al. 1995. 180-kD bullous pemphigoid antigen (BP180) is deficient in generalized atrophic benign epidermolysis bullosa. J. Clin. Invest. 95:1345-1352.

16. McGrath, J.A., et al. 1995. Mutations in the $180-\mathrm{kD}$ bullous pemphigoid antigen (BPAG2), a transmembrane hemidesmosomal collagen (COL17A1), in generalized atrophic benign epidermolysis bullosa. Nat. Genet. 11:83-86.

17. McGrath, J.A., et al. 1996. A homozygous deletion mutation in the gene for the $180-\mathrm{kDa}$ bullous pemphigoid antigen (BPAG2) in a family with generalized atrophic benign epidermolysis bullosa. J. Invest. Dermatol. 106:771-774.

18. Darling, T.N., et al. 1997. Premature termination codons are present on both alleles of the bullous pemphigoid antigen 2/type XVII collagen gene in five Austrian families with generalized atrophic benign epidermolysis bullosa. J. Invest. Dermatol. 108:463-468.

19. Emmert-Buck, M.R., et al. 1996. Laser capture microdissection. Science. 274:998-1001.

20. Bonner, R.F., et al. 1997. Laser capture microdissection: molecular analysis of tissue. Science. 278:1481-1483.

21. Hintner, H., and Wolff, K. 1982. Generalized atrophic benign epidermolysis bullosa. Arch. Dermatol. 118:375-384.

22. Darling, T.N., et al. 1998. A deletion mutation in COL17A1 in five Austrian families with generalized atrophic benign epidermolysis bullosa represents propagation of an ancestral allele. J. Invest. Dermatol. 110:170-173.

23. Masunaga, T., et al. 1997. The extracellular domain of BPAG2 localizes to anchoring filaments and its carboxyl terminus extends to the lamina densa of normal human epidermal basement membrane. J. Invest. Dermatol. 109:200-206.

24. Stanley, J.R., Hawley-Nelson, P., Yuspa, S.H., Shevach, E.F., and Katz, S.I. 1981. Characterization of bullous pemphigoid antigen: a unique basement membrane protein of stratified squamous epithelia. Cell. 24:897-903.

25. Basset-Seguin, N., Caughman, S.W., and Yancey, K.B. 1990. A-431 cells and human keratinocytes synthesize and secrete the third component of complement. J. Invest. Dermatol. 95:621-625.

26. Greene, C.N., and Jinks-Robertson, S. 1997. Frameshift intermediates in homopolymer runs are removed efficiently by yeast mismatch repair proteins. Mol. Cell. Biol. 17:2844-2850.

27. McGrath, J.A., et al. 1996. Compound heterozygosity for a dominant glycine substitution and a recessive internal duplication mutation in the type XVII collagen gene results in junctional epidermolysis bullosa and abnormal dentition. Am. J. Pathol. 148:1787-1796.

28. Fanin, M., et al. 1995. Dystrophin-positive fibers in Duchenne dystrophy: origin and correlation to clinical course. Muscle Nerve. 18:1115-1120. 